> La trypanosomiase humaine africaine, ou maladie du sommeil, demeure un problème préoccupant en Afrique intertropicale. Cette maladie nécessite une surveillance systématique des populations, particulièrement pour la trypanosomiase à Trypanosoma brucei gambiense, qui présente une longue période asymptomatique. En l'absence de signes cliniques spécifiques, le dépistage exhaustif des populations demeure I'unique moyen de contrôler cette maladie et d'éviter sa propagation. Le manque de sensibilité et de spécificité des tests diagnostiques classiques a conduit, ces dernières années, à l'utilisation des outils moléculaires. L'amplification par PCR de séquences spécifiques des parasites a considérablement amélioré le diagnostic de l'infection, le diagnostic de phase de la maladie, ainsi que le suivi post-thérapeutique. Cependant, des limitations à une application en routine existent et des recherches sont encore nécessaires pour faire de cette technique un réel outil de contrôle et de lutte contre la maladie du sommeil. <

\section{Apports et limites du diagnostic de la trypanosomiase humaine africaine}

\author{
Vincent Jamonneau, Philippe Solano, \\ Mathurin Koffi, Mélanie Denizot, Gérard Cuny
}

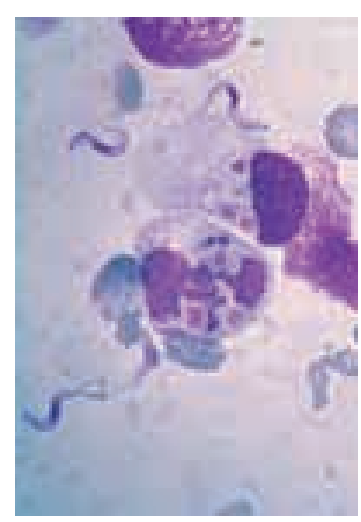

Dans le cas de la forme gambienne, le cycle de transmission hommemouche-homme semble prédominer. L'existence d'un réservoir animal a été mise en évidence [2]. Son importance épidémiologique dans la transmission et la persistance de la maladie est toutefois encore mal connue. La forme rhodésienne est une zoonose typique. La transmission à l'homme (souvent considéré comme hôte accidentel) se fait suivant le cycle animal sauvage ou domestique-mouche-homme. Cette différence entre les deux formes de la maladie conditionne les stratégies de lutte : dans la forme gambienne, le traitement de tous les malades, associé à une lutte antivectorielle, devrait théoriquement permettre une éradication de la maladie. Cependant le traitement de tous les malades nécessite un dépistage exhaustif.

La THA évolue en deux phases, une première période, lymphatico-sanguine, durant laquelle le parasite se multiplie dans le sang et dans la lymphe, et une deuxième période, méningo-encéphalitique qui correspond au passage du parasite dans le liquide céphalorachidien (LCR). Au cours de la première période (qui peut durer plusieurs années dans la forme gambienne, 
mais seulement quelques semaines dans la forme rhodésienne), le malade présente des signes cliniques non spécifiques tels que des accès de fièvre fréquents, des céphalées ou des signes cutanés [3]. La phase méningo-encéphalitique se caractérise habituellement par l'apparition de troubles neurologiques (troubles du comportement, de la conscience, de la motricité), qui, sans traitement, conduisent au décès du malade. Les médicaments utilisés en première période (pentamidine, suramine) sont considérés comme peu toxiques, alors que le mélarsoprol, utilisé dans la seconde période, est un dérivé arsenical ayant des effets secondaires importants, responsable d'environ $10 \%$ d'encéphalopathies réactives chez les patients traités.

Le diagnostic clinique de THA étant difficile $[3,4]$, des méthodes de diagnostic direct ou indirect sont nécessaires. Le diagnostic direct vise à rechercher la présence du parasite dans les liquides biologiques, alors que les méthodes indirectes mettent en évidence l'infection parasitaire à l'aide de tests sérologiques. Le diagnostic indirect est utilisé pour déterminer les sujets sur lesquels porteront les examens du diagnostic direct.

Les méthodes de diagnostic indirect utilisées en dépistage de routine se fondent sur la détection d'anticorps spécifiques par les tests CATT (card agglutination test for trypanosomiasis) Tb gambiense [5] et Latex Tb gambiense [6]. En cas de positivité de l'un de ces tests, le parasite devra être mis en évidence par une méthode directe pour permettre le traitement du malade [1]. Le diagnostic direct consiste en l'observation microscopique de la lymphe et du sang. Si des adénopathies cervicales sont présentes, l'observation de la lymphe se fait simplement par examen direct du suc ganglionnaire. En raison des parasitémies souvent très faibles, l'observation du sang nécessite l'utilisation de techniques de concentration comme la centrifugation en tubes capillaires (CTC) [7], la colonne échangeuse d'anions (mAECt) [8], ou le $\mathrm{PBC}$ (quantitative buffy-coat) [9] (Figure 1).

L'examen du LCR permet de diagnostiquer la phase de la maladie. La recherche de trypanosomes se fait clas- siquement par simple (SC) ou double (DC) centrifugation du LCR $[10,11]$. Ces techniques étant peu sensibles, des critères indirects d'altération du LCR sont aussi utilisés, comme l'augmentation de la protéinorachie (dosage des protéines) et de la cytorachie (comptage des leucocytes). Les valeurs préconisées par l'Organisation Mondiale de la Santé [1] pour établir le diagnostic de phase sont indiquées dans la Figure 1.

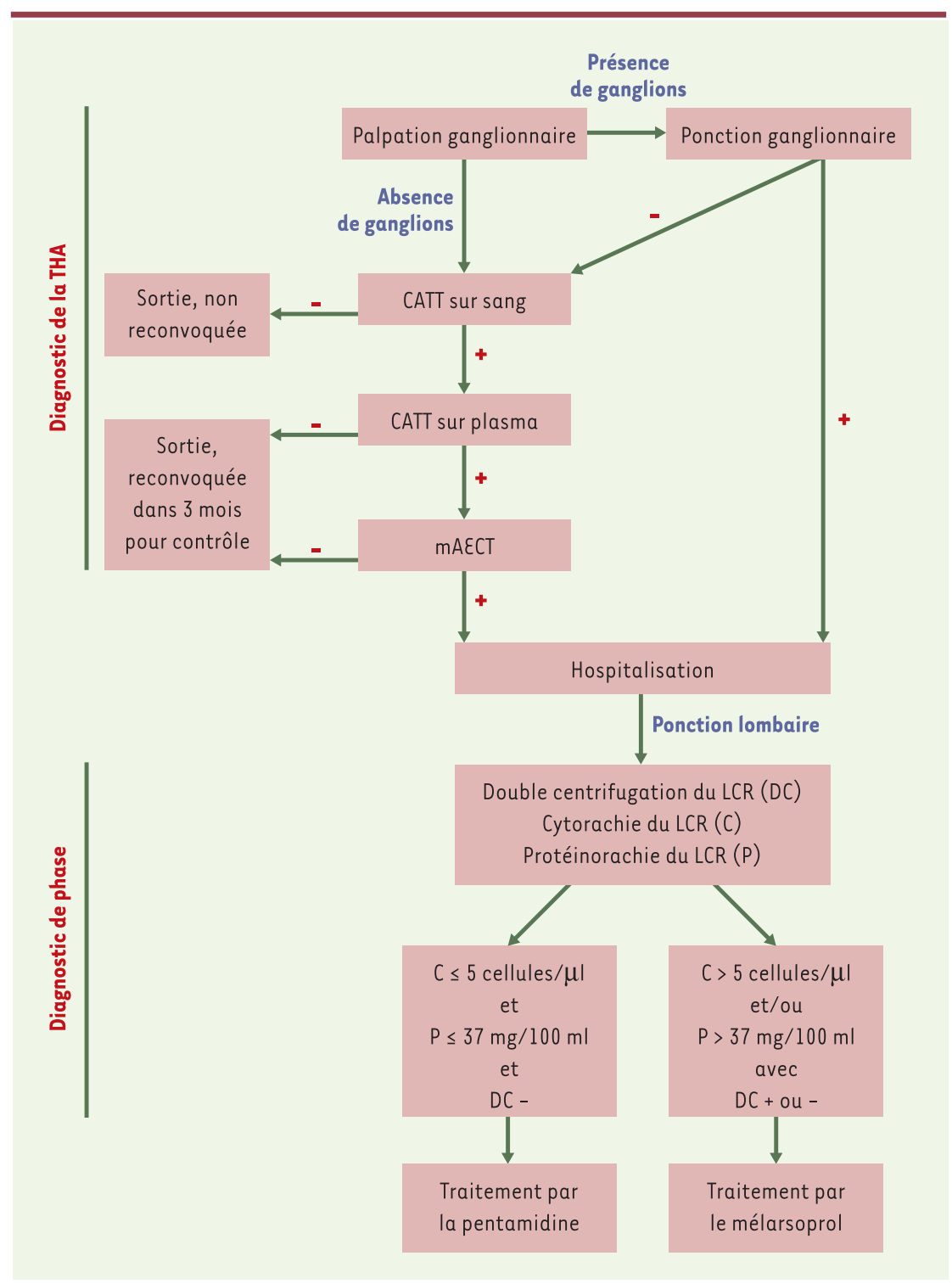

Figure 1. Dépistage et diagnostic de phase de la trypanosomiase humaine africaine. Le sujet est hospitalisé si la recherche de trypanosomes dans les adénopathies est positive ou si un test CATT (card agglutination test for trypanosomiasis) sanguin et plasmatique ainsi qu'une recherche de parasites après concentration sur colonne échangeuse d'anions (mAECT) sont positifs. Le diagnostic de phase de la maladie repose sur l'analyse du liquide céphalorachidien (LCR), après double centrifugation (DC), à la recherche de trypanosomes et d'une protéinorachie ou d'une cytorachie anormales. Le passage du parasite dans le LCR signe l'atteinte méningo-encéphalitique qui impliquerait un traitement par le mélarsoprol. 


\section{Améliorer le diagnostic et la détermination du stade de la maladie}

Le diagnostic et la détermination de la phase de la maladie sont d'une importance primordiale: (1) au niveau individuel, car ils permettent d'assigner un statut de «malade» à la personne examinée et de décider du traitement selon la phase de la maladie; (2) au niveau de la population, car les stratégies de lutte contre cette maladie, qui touchent des populations rurales dans des régions souvent difficiles d'accès, reposent en partie sur la détection précoce et le traitement des malades. Chaque méthode de diagnostic est évaluée en fonction de sa sensibilité (vrais positifs/vrais positifs + faux négatifs) et de sa spécificité (vrais négatifs/vrais négatifs + faux positifs) [12]. Les techniques actuellement utilisées manquent souvent de sensibilité et/ou de spécificité : défaut de sensibilité des examens parasitologiques du fait de parasitémies faibles [13]; défaut de sensibilité des méthodes indirectes dans le cas d'infections précoces (les anticorps ou antigènes recherchés ne circulent pas encore dans le sang) ou d'infections ne produisant pas les anticorps ou antigènes recherchés [14]; défaut de spécificité des méthodes sérologiques souvent attribué à des réactions croisées avec d'autres trypanosomes ou d'autres parasites humains, à la persistance d'anticorps ou d'antigènes durant plusieurs mois, voire plusieurs années après traitement [15].

\section{Apports de l'outil moléculaire}

Depuis ces dernières années, la détection de l'ADN de trypanosomes par PCR (polymerase chain reaction) est évaluée pour améliorer le diagnostic et la détermination du stade de la THA. Plusieurs amorces oligonucléotidiques ont été testées sur le sang ou sur LCR [16-23]. Quel que soit le protocole utilisé, la PCR semble être généralement beaucoup plus sensible que les techniques parasitologiques classiques, avec une limite de détection allant de 1 à 40 trypanosomes par $\mathrm{ml}$ de sang. En comparaison, la mAECt, considérée actuellement comme l'examen parasitologique le plus sensible, donne une limite de détection de 100 trypanosomes par $\mathrm{ml}$ de sang [1, 8]. À la différence des techniques sérologiques, la PCR permet de détecter précocement les infections trypanosomiennes [24]. Appliquée au LCR, la PCR s'est avérée plus sensible que la double centrifugation et semble également permettre de diagnostiquer très précocement les rechutes, à la différence des techniques classiquement utilisées [19].

Les amorces oligonucléotidiques couramment utilisées, spécifiques de $T$. brucei sl, permettent d'éviter les fausses positivités dues à d'autres parasitoses (filarioses, paludisme) ou à la présence transitoire d'autres trypanosomes (trypanosomes animaux). Ces avantages feraient de la PCR un test spécifique. À la différence des techniques indirectes qui peuvent rester positives plusieurs années après le traitement (persistance des anticorps ou des antigènes dans le sang), l'observation d'une PCR positive représenterait réellement une infection active, la persistance de l'ADN de trypanosomes morts dans le sang n'excédant pas un à deux jours [24].

\section{Limites rencontrées}

Plusieurs auteurs ont rapporté des discordances entre les résultats des tests sérologiques et ceux obtenus par PCR. Le CATT, utilisé pour le dépistage de masse, manque de spécificité ; un examen parasitologique est donc réalisé sur les sujets positifs pour rechercher les trypanosomes dans les liquides biologiques. Des études menées en Ouganda rapportent une absence significative de corrélation entre le CATT sur sang et la $P C R$ [20]. Des problèmes de reproductibilité de la PCR à partir de sang ont été mis en évidence en Côte d'Ivoire [25]. Chez des sujets séropositifs ayant un examen parasitologique négatif, deux PCR successives ne donnent pas le même résultat. Le suivi longitudinal de ces cas montre que des résultats PCR positifs apparaissent moins souvent dans une population de séropositifs (définis comme positifs en sérologie à chacune des six visites du suivi) que dans une population de séronégatifs (définis comme négatifs en sérologie à au moins l'une des six visites) [26]. Enfin, sur des échantillons de sang de sujets séropositifs non confirmés à l'examen parasitologique, des discordances sont trouvées en fonction des méthodes d'extraction de I'ADN [17].

La spécificité de la technique a également été remise en cause car les amorces les plus utilisées amplifient les souches du groupe de $T$. brucei sl comprenant, outre $T b$ gambiense et $T b$ rhodesiense, Trypanosoma brucei brucei qui n'est pas pathogène pour l'homme. Ces trypanosomes non pathogènes pourraient induire des faux positifs [26]. La spécificité des amorces oligonucléotidiques conventionnelles à $T$. brucei sl [16] a même été contestée, des réactions croisées avec $T$. vivax et $T$. congolense ayant été décrites [22]. Une observation récente pourrait également expliquer en partie l'existence de fauxpositifs : des PCR in situ ont montré que des séquences de trypanosomes étaient insérées dans les tissus (macrophages) d'un rat infecté par $T$. brucei brucei, traité à la pentamidine [22]. Si elle était confirmée, cette observation pourrait avoir des implications très importantes en termes de diagnostic par PCR et d'interactions hôte-parasite.

Concernant le diagnostic de phase de la maladie, la positivité de la PCR dans le LCR signe la présence du parasite dans le LCR et impliquerait de traiter au mélarsoprol tous les sujets positifs. Or, dans une étude récente menée en Côte d'Ivoire, plusieurs sujets dont la PCR du LCR était positive, mais la DC négative et le nombre de cellules compris entre 0 et 5 , ont été traités efficacement par la pentamidine (voir Figure 1) [27]. De plus, des sujets ayant entre 0 et 20 cellules/ $\mu$ l ou positifs en DC ont pu être traités efficacement par la pentamidine, ce qui laisse penser que 
même la présence de trypanosomes dans le LCR ne semble pas justifier l'emploi du mélarsoprol [28]. Même si la PCR/LCR permet d'améliorer le diagnostic de phase de la maladie, son intérêt n'apparaît pas toujours évident dans la décision thérapeutique.

\section{Conclusions et perspectives}

L'utilisation de la PCR dans le dépistage et le diagnostic de phase de la THA pourrait apporter des avancées importantes. Dans le cadre du dépistage actif, la PCR utilisée en aval des tests sérologiques permettrait d'établir si un sujet est infecté, avec une sensibilité apparemment supérieure aux méthodes classiquement utilisées. Mais tant que l'on ne pourra pas affirmer avec certitude qu'une PCR positive est le signe d'une infection active, l'utilisation de cette méthode dans le cadre du dépistage restera marginale. La PCR offre une sensibilité apparemment inégalée et une spécificité correcte, mais les résultats obtenus chez des sujets ayant un test CATT positif non confirmé par l'examen parasitologique sont controversés. Cependant, le problème du diagnostic chez ces sujets ne concerne pas seulement la PCR, mais toutes les techniques de diagnostic.

En termes d'application sur le LCR, la PCR peut permettre de déterminer si un malade est en première ou seconde phase, ce qui conditionnera son traitement. Mais a-t-on réellement intérêt à utiliser le test le plus sensible pour le diagnostic de phase, alors que plusieurs travaux rapportent que l'on peut traiter avec succès des malades en seconde phase par la pentamidine [28]. $\varepsilon n$ revanche, la PCR présente un intérêt certain dans le suivi du traitement et le diagnostic précoce des rechutes, la mise en évidence d'ADN trypanosomien dans le LCR pouvant être considérée comme la signature d'une réapparition de la maladie.

Dans toutes ces applications, un pas décisif aura été franchi lorsque des amorces spécifiques de I'ADN de trypanosomes pathogènes pour l'homme seront disponibles. Des candidats potentiels sont actuellement à l'étude pour le diagnostic moléculaire de la THA : SRA (serum resistant associated gene) pour Tb rhodesiense [29] ; TgsGP (T gambiense specific glycoprotein) pour Tb gambiense [23] ; des séquences d'ADN microsatellites spécifiques de Tb gambiense qui différencient apparemment les trypanosomes pathogènes de l'homme des autres $T$. brucei [30]. Ces marqueurs pourraient résoudre les problèmes de spécificité de la PCR, à condition de conserver des sensibilités équivalentes à celles obtenues avec les amorces oligonucléotidiques utilisées actuellement.

La complexité des processus mis en jeu lors du passage du trypanosome dans le sang et dans le LCR est due à des phénomènes liés au parasite (variabilité génétique, différence de virulence) ou à l'hôte (sensibilité individuelle à l'infection, processus d'altération du système nerveux central). Cette complexité peut affecter la fiabilité des tests de dépistage et de diagnostic de phase de la THA et rendre difficile la décision thérapeutique (doit-on traiter un sujet ? Avec quel médica- ment ?). Même si la PCR peut contribuer au dépistage et au diagnostic de phase, la mise au point d'un outil fiable, applicable à tous les sujets atteints de THA, et permettant de prendre la meilleure décision thérapeutique nécessite une meilleure connaissance de ces phénomènes. L'élaboration d'outils novateurs et de stratégies thérapeutiques nouvelles non toxiques aidera à lutter efficacement contre cette maladie, surtout à l'heure où les échecs thérapeutiques par résistance au traitement se multiplient [1]. $\diamond$

\section{SUMMARY}

\section{Contributions and limits of the diagnosis}

of human African trypanosomiasis

Human African trypanosomiasis, or sleeping sickness, is still a worrying problem in Africa. Sleeping sickness is a disease for which a systematic monitoring is necessary, particularly for the trypanosomiasis caused by Trypanosoma brucei gambiense, which is characterized by a long asymptomatic stage. In the absence of specific clinical signs, mass screening of populations remains the only way to control the disease and to avoid its spreading. The lack of sensitivity and specificity of the diagnosis tests classically used led to the developpement of molecular tools. PCR amplification of parasite specific sequences has considerably improved the diagnostic of the parasitic infection, the stage diagnosis as well as the post-therapeutic follow-up. But there are limits with a use in routine and research is still necessary to make PCR a real tool for control of sleeping sickness. $\bullet$

\section{RÉFÉRENCES}

1. Anonymous. Control and surveillance of African trypanosomiasis. Report of a WHO expert committee. World Health Org Tech Rep Ser 1998 ; 881 : 1-114.

2. Mehlitz D, Zillmann U, Scott C, et al. Epidemiological studies on the animal reservoir of Gambiense sleeping sickness. Part III. Characterization of trypanozoon stocks by isoenzymes and sensitivity to human serum. Tropen Med Parasitol 1982 ; $33: 113-8$.

3. Jannin J, Moulia-Pelat JP, Chanfreau B, et al. Trypanosomiase humaine africaine : étude d'un score de présomption de diagnostic au Congo. B World Health Org 1993 ; $71: 215-22$

4. Jamonneau V, Garcia A, N'Guessan P, et al. Clinical and biological evolution of human African trypanosomiasis in Côte d'Ivoire. Ann Trop Med Parasitol 2000 ; $94: 831-5$.

5. Magnus $\varepsilon$, Vervoort T, Van Meirvenne N. A card-agglutination test with stained trypanosomes (CATT) for the serological diagnosis of $T$. gambiense trypanosomiasis. Ann Soc Belg Med Trop 1978 ; 59 : 169-76.

6. Buscher $P$, Draelants $\varepsilon$, Magnus $\varepsilon$, et al. An experimental latex agglutination test for antibody detection in humain African trypanosomiasis. Ann Soc Belg Med Trop $1991 ; 71: 267-73$.

7. Woo PTK. The haematocrit centrifuge technique for the diagnosis of African trypanosomiasis. Acta Trop $1970 ; 27: 384-6$.

8. Lumsden WHR, Kimber CD, Strange M. Trypanosoma brucei : a miniature anionexchange/centrifugation technique for the detection of low parasitemias in mice. Trans Roy Soc Trop Med Hyg 1977 ; 71 : $421-4$.

9. Bailey JW, Smith DH. The use of the acridine orange $Q B C$ technique in the diagnosis of African trypanosomiasis. Trans Roy Soc Trop Med Hyg 1992; 86 : 630.

10. Miezan TW, Meda HA, Doua F, et al. Simple centrifugation of cerebrospinal fluid in a sealed Pasteur pipette for simple, rapid and sensisitive detection of trypanosomes. Trans Roy Soc Trop Med Hyg $2000 ; 94: 293$. 
11. Cattand P, Miezan TW, de Raadt P. Human African trypanosomiasis : use of double centrifugation of cerebrospinal fluid to detect trypanosomes. $B$ World Health Org $1988 ; 66: 83-6$.

12. Dukes $P$, Rickman LR, Killick-Kendrick $R$, et al. A field comparison of seven diagnostic techniques for human African trypanosomiasis in the Luangwa valley, Zambia. Tropen Med Parasitol 1984 ; 35 : 141-7.

13. Truc P, Jamonneau V, N'Guessan P, et al. Parasitological diagnosis of African trypanosomiasis : a comparison of the $\mathrm{QBC}{ }^{\circledR}$ and miniature anion-exchange centrifugation techniques. Trans Roy Soc Trop Med Hyg 1998 ; 92 : 288-9.

14. Dukes P, Gibson WC, Gashumba JK, et al. Absence of the LiTat 1.3 (CATT antigen) gene in Trypanosoma brucei gambiense stocks from Cameroon. Acta Trop 1992 ; $51: 123-4$.

15. Diallo PB, Truc P, Méda HA, Kamenan A. Diagnostic sérologique de la trypanosomiase humaine africaine à Trypanosoma brucei gambiense. 1. Obtention et utilisation d'antigènes bruts dans les tests ELISA et d'agglutination au latex. Bull Soc Pathol Exot 1996 ; 89 : 262-8.

16. Moser DR, Cook GA, Ochs DE, et al. Detection of Trypanosoma congolense and Trypanosoma brucei subspecies by DNA amplification using the polymerase chain reaction. Parasitology $1989 ; 99: 57-66$

17. Kanmogne GD, Stevens JR, Asonganyi T, Gibson WC. Genetic heterogeneity in the Trypanosoma brucei gambiense genome analysed by random amplification of polymorphic DNA. Parasitol Res $1996 ; 82$ : 535-41.

18. Penchenier L, Dumas V, Grébaut $P$, et al. Improvement of blood and fly gut processing for PCR diagnosis of trypanosomiasis. Parasite $1996 ; 4: 387-9$

19. Truc $P$, Jamonneau V, Cuny $G$, Frezil JL. Use of polymerase chain reaction in human African trypanosomiasis stage determination and follow-up. B World Health Org $1999 ; 77$ : 745-9.

20. Kyambadde JW, Enyaru JCK, Matovu $\varepsilon$, et al. Detection of trypanosomes in suspected sleeping sickness patients in Uganda using the polymerase chain reaction. B World Health Org $2000 ; 78$ : 119-24.

21. Kabiri M, Franco JR, Simarro PP, et al. Detection of Trypanosoma brucei gambiense in sleeping sickness suspects by PCR amplification of expression-site-associated genes 6 and 7. Trop Med Int Health 1999; $10: 658-61$.
22. De Almeida PP, Ndao M, Van Meirvenne N, Geerts S. Diagnostic evaluation of PCR on dried blood samples from goats experimentally infected with Trypanosoma brucei brucei. Acta Trop $1998 ; 70: 269-76$.

23. Radwanska M, Chamekh M, Vanhamme L, et al. The serum resistance-associated gene as a diagnostic tool for the detection of Trypanosoma brucei rhodesiense. Am J Trop Med Hyg 2002 ; $67: 684-90$.

24. Bengaly Z, Kasbari M, Desquesnes M, Sidibé I. Validation of a polymerase chain reaction assay for monitoring the therapeutic efficacy of diminazene aceturate in trypanosome-infected sheep. Vet Parasitol 2001; 96 : 101-13.

25. Solano P, Jamonneau V, N'Guessan P, et al. Comparison of different DNA preparation protocols for PCR diagnosis of human trypanosomiasis. Acta Trop $2002 ; 82: 349-56$

26. Garcia A, Jamonneau V, Magnus $\varepsilon$, et al. Longitudinal survey of positive card agglutination trypanosomiasis test (CATT) but apparently aparasitemic individuals in Côte d'Ivoire : evidence for complex and heterogeneous population. Trop Med Int Health $2000 ; 5: 786-93$

27. Jamonneau V, Solano P, Garcia A, et al. Stage determination and therapeutic decision in human African trypanosomosis : value of PCR and IgM quantification on the cerebrospinal fluid of sleeping sickness patients in Côte d'Ivoire. Trop Med Int Health $2003 ; 8: 1-6$

28. Doua F, Miezan TW, Sano JR, et al. The efficacy of pentamidine in the treatment of early-late stage Trypanosoma brucei gambiense trypanosomiasis. Am J Trop Med Hyg $1996 ; 55: 586-8$.

29. Gibson W. Will the real Trypanosoma brucei rhodesiense please step forward ? Trends Parasitol $2002 ; 18: 486-90$.

30. Biteau N, Bringaud F, Gibson WC, et al. Characterisation of trypanozoon isolates using micro and minisatellite markers. Mol Biochem Parasitol $2000 ; 105: 185-201$.

\section{UN OUVRAGE EXCEPTIONNEL QUI REND COMPTE DES PROGRES LES PLUS RÉCENTS REALISÉS EN GENEETIQUE}

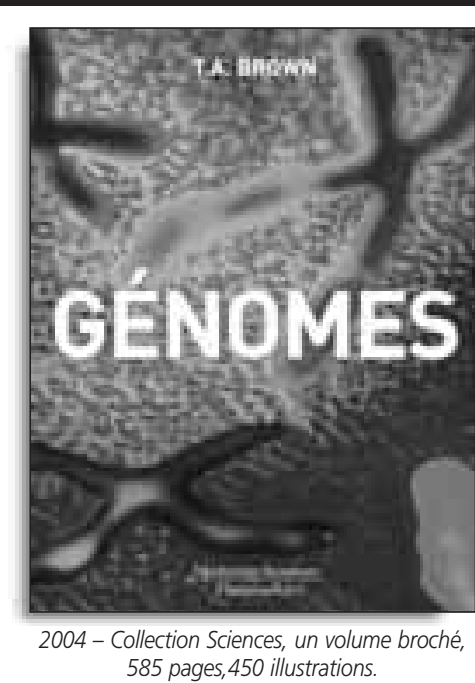

GÉNOMES Terry A. BROWN

traduction francaise d'Irène Mowszowicz, Service de biochimie, Hôpital Necker-Enfants Malades, Paris. D'extraordinaires progrès ont été réalisés en génétique ces toutes dernières années, en particulier le séquençage complet du génome de centaines d'organismes, des bactéries à l'homme, qui a révolutionné notre compréhension des êtres vivants et de leurs interrelations. Cet ouvrage exceptionnel en rend compte. II est divisé en 4 parties • le génome humain et les transcriptions • l'étude du génome, cartographie et séquençage $\bullet$ le fonctionnement du génome, la synthèse du arn $\bullet$ la régulation et l'activité du génome $\bullet$ les modalités de réplication du génome, le clonage et les applications notamment médicales. L'auteur s'appuie sur une pédagogie efficace et propose des listes de notions importantes, des encadrés avec les points-clés, des notes techniques, de nombreuses figures en 5 couleurs très clairement commentées, un bilan sur l'état de la recherche dans le cadre de la question traitée, etc. Des séries de questions et problèmes à la fin de chaque chapitre permettent de s'auto-évaluer. L'ouvrage se termine par un glossaire de 1200 termes.

Au total un livre unique de 585 pages et 450 illustrations en couleurs, indispensable à l'étudiant comme à l'enseignant pour apprendre et comprendre la génétique moderne, et au chercheur pour disposer d'un outil actuel.

En vente chez votre libraire spécialisé, sur notre site www.medecine.flammarion.com ou par correspondance BON DE COMMANDE à retourner à 